\title{
Homotopy characterization of weakly flat knots
}

by

Vo-Thanh-Liem* (Athens, Ga)

Abstract. Let $X$ be a compactum in the $n$-sphere $S^{n}, n \geqslant 6$, having the shape of $S^{n-2}$. If $X$ is globally homotopically unknotted in $S^{n}$, then its complement is PL-homeomorphic to the complement of a locally flat codimension-2 sphere.

1. Introduction. After solving Siebenmann's conjecture that "a globally 1-alg codimension-2 sphere in $S^{n}, n \geqslant 5$, whose complement has the homotopy type of $S^{1}$, is weakly flat", Hollingsworth and Rushing raised in [4] the following conjecture: "Assume that $X$ is a globally homotopically unknotted codimension-2 sphere in $S^{n}$, then there is a locally flat $(n-2)$-sphere $\Sigma$ in $S^{n}$ such that $S^{n}-X \approx S^{n}-\Sigma "$

In [3], Daverman gives a "yes" answer to that conjecture for shape clas of $S^{n-2}$ if $S^{n}-X$ has the homotopy type of a finite complex.

As it is stated in the abstract, it will be shown in this paper that the conjecture is true for the embedding of the shape class of the codimension- 2 sphere for $n \geqslant 6$ by proving, through Lemmas $1-6$, that the end of $S^{n}-X$ has arbitrarily small, finitely dominated neighborhoods.

Actually, a little more general results, Theorem II-III are obtained. In order to make the paper readable, however, the proof for a simple case is presented in detail and then, an outline for the general case is given.

Finally, the author wishes to express his gratitude to J. G. Hollingsworth for his interesting discussions.

\section{Notations and definitions}

f.g. finitely generated,

$\stackrel{\mathrm{PL}}{\approx} \quad$ (PL-) homeomorphic or isomorphic,

$\simeq \quad$ homotopy equivalence or homotopic,

$\sim$ homologous,

$\partial V$, Int $V$ boundary, interior of a manifold $V$,

$i, j \quad$ inclusion map;

* The author is receiving a postdoctoral fellowship at University of Georgia. 
$f_{\text {\# }}, f_{*} \quad$ induced map on homotopy, homology groups,

$H_{*}\left(H^{*}\right)$ singular (co-) homology with $Z$ or $\Lambda$-coefficient where $\Lambda$ is the integral group ring $Z\left(\pi_{1}\right)$,

1. $[x] \quad \Lambda$-submodule generated by $x$,

$\tilde{V} \quad$ universal covering space of the manifold $V$,

$D^{k} \quad k$-dimensional PL-ball.

For basic shape theory results we shall refer to [1] and [9]. For convenience, both shape theories in [1] and [9] will be used as a justification for [10].

Let $X$ be a compactum in a manifold $M . X$ is globally 1 -alg in $M$ if for every neighborhood $U$ of $X$ in $M$ there is a neighborhood $V$ of $X$ in $U$ such-that $f: S^{1} \rightarrow V-X, f \sim 0$ in $V-X$, then $f \simeq 0$ in $U-X . X$ is globally $k-$ LC in $M$ if for every neighborhood $U$ of $X$ in $M$, there is a neighborhood $V$ of $X$ in $U$ such that $i_{\#}: \pi_{k}(V-X) \rightarrow \pi_{k}(U-X)$ is trivial. $X$ is globally homotopically unknotted in $M$ if it is globally 1 -alg and globally $k-L C$, for $2 \leqslant k \leqslant\left[\frac{1}{2} \operatorname{dim} M\right]$, where $\left[\frac{1}{2} \operatorname{dim} M\right]$ is the integral part of $\frac{1}{2} \operatorname{dim} M$

For definitions of the end of a non-compact manifold, stable end $\varepsilon, \pi_{1}(\varepsilon)$, etc... we refer to [11].

For definitions of regular neighborhood, PL-embedding, PL-homeomorphism, etc... we refer to Hudson [5].

An open manifold $\Omega^{n}$ with one end has a collar at the end if $\Omega$ has a submanifold $W^{n}$ such that $\Omega$-Int $W$ is compact and $W \approx \partial W \times[0,1)$. $\subset$ Int $V$

PL (or DIFF)-embedding $\varphi:\left(D^{k+1}, S^{k}\right) \rightarrow(V, \partial V)$ is nice if $\varphi\left(\ln t D^{k+1}\right)$

$S_{1}^{1} \vee \ldots \vee S_{\mu}^{1}$ denotes the we,dge product of $\mu$ copies of the circle $S^{1}$. Let $K$ be a finite subcomplex of a PL-manifold. $N(K, M)$ denotes the regular
neighborhood of $K$ in $M$ (see [5]).

3. Results and details of the proof. Throughout Lemmas 1-6, $X$ denotes a compactum in $S^{n}$ having the shape of the codimension-2, sphere $S^{n-2}$.

LEMMA 1. If $X$ is globally 1 -alg, then there are arbitrarily small, simply connected, PL-manifold neighborhood $V$ 's of $X$ in $S^{n}$ such that $V-X$ is a 1 -neighborhood of the
end of $S^{n}-X$, i.e.

(*) (i) $\partial V, V-X$ are path connected,

(ii) $\pi_{1}(\partial V) \rightarrow \pi_{1}(V-X)$ induced by the inclusion map is an isomorphism.

Proof. Since $X$ has the shape of $S^{n-2}, S^{n}-X$ is a homology 1 -sphere with one end $\varepsilon$. According to Lemma 1 of [3] or Lemma 8 below, the end of $S^{\prime \prime}-X$ is stable and $\pi_{1}(\varepsilon)=Z$, a finitely presented group. Hence, Theorem 3.10 in [II] shows that the end $\varepsilon$ has arbitrarily small neighborhood $W$ 's.

Let $V=W \cup X$. It suffices to show that $V$ is simply connected. Since $\operatorname{Sh}(X)=\operatorname{Sh}\left(S^{n-2}\right)$ and $S^{n-2}$ is simply connected $(n \geqslant 5)$, there is a $W^{\prime} \subset W$ such that $W^{\prime}$ is a 1 -neighborhood of the end and the map $\pi_{1}\left(V^{\prime}\right) \rightarrow \pi_{1}(V)$ is trivial,
where $V^{\prime}=W^{\prime} \cup X$.
Now, since the map $\pi_{1}\left(W^{\prime}\right) \rightarrow \pi_{1}(W)$ is an isomorphism (Lemma 3.12 [11]), a loop in $W$-Int $W^{\prime}=V$-Int $V^{\prime}$ is homotopic in $W \subset V$ to a loop in $W^{\prime} \subset V^{\prime}$. Therefore, the map $\pi_{1}\left(V-\operatorname{Int} V^{\prime}\right) \rightarrow \pi_{1}(V)$ is trivial. Then, $\pi_{1}(V)=0$ by Van Kampen's theorem.

Lemma 2. Let $V$ be a neightrorhood of $X$ as in Lemma 1. Then $\pi_{2}(V-X)$ and $\pi_{2}(V-X, \partial V)$ cire f.g. A-modules.

Proof. Let $p: \widetilde{V-X} \rightarrow V-X$ be the universal covering space of $V-X$. Let $W$ be a neighborhood of $X$ in $\operatorname{Int} V$ as in Lemma 1 such that $\operatorname{Im}\left(i_{\text {吸 }}: \pi_{2}(W-X) \rightarrow \pi_{2}(V-X)\right)$ is trivial ( $X$ is globally $2-\mathrm{LC}$ ). Then, the restrictions of $p$ on, $p^{-1}(\partial V)$, $p^{-1}(W-X), p^{-1}(\partial W)$ are also universal covering projections since the corresponding inclusion maps from $\partial V, W-X, \partial W$ into $V-X$ induce isomorphisms on their fundamental groups.

Let $A=V$-Int $W$. Then, $p \mid p^{-1}(A): p^{-1}(A) \rightarrow A$ is a covering map. Moreover $p^{-1}(A)$ is a simply connected since it is a manifold with simply connected boundary $p^{-1}(\partial V \cup \partial W)$ and it is contained in the simply connected manifold $\overparen{V-X}$. Hence, $p^{-1}(A) \rightarrow A$ is also the universal covering space of $A$.

Remark 1. Let $e_{0}$ be a fixed point of the fiber $p^{-1}\left(x_{0}\right)$, where $x_{0} \in \partial W$. It is known that if we assign to each element $h$ of the covering transformation group $G$ of $p^{-1}(A)$ the element $[\alpha] \in \pi_{1}\left(A, x_{0}\right)$ such that $\tilde{\alpha}_{e_{0}}(1)=h\left(e_{0}\right)$, where $\tilde{\alpha}_{e_{0}}$ is the lift of $\alpha$ starting at $e_{0}$, then we define an isomorphism between $G$ and $\pi_{1}\left(A, x_{0}\right)$. Using this fact, we can prove that $i_{\text {非 }}: \pi_{1}\left(\partial W, x_{0}\right) \rightarrow \pi_{1}\left(A, x_{0}\right)$ is an isomorphism.

Therefore, we can always consider the homology of universal covering of spaces, pairs as $\Lambda$-modules induced by the same covering transformation group isomorphic to $\pi_{1}(\varepsilon) \approx Z$.

Now, we return to the proof of the lemma. The Mayer-Vietoris sequence of the triple $\overparen{(V-X}, \tilde{A}, \overparen{W-X})$ gives the exact sequence

$$
H_{2}(\tilde{A}) \oplus H_{2}(\widetilde{W-X}) \rightarrow H_{2}(\widetilde{V-X}) \rightarrow H_{1}(\widetilde{\partial W}) .
$$

Hence, we have the exact sequence

$$
\pi_{2}(A) \oplus \pi_{2}(W-X) \rightarrow \pi_{2}(V-X) \rightarrow 0,
$$

by Hurewicz's Theorem.

SublFmma A. Let $A$ be a finite complex such that $\pi_{1}(A)$ is a free group of $\mu$ generators. If $\pi_{q}(A)=0$ for $2 \leqslant q \leqslant m-1$, then $\pi_{m}(A)$ is a f.g. Z $Z \pi_{1}-$ module.

Proof. Let $f: S_{1}^{1} \vee \ldots \vee S_{\mu}^{1} \rightarrow A$ be an imbedding inducing an isomorphism on the fundamental groups. Then, it is clear that $f$ is $(m-1)$-connected since $\pi_{q}(A)=0$, $q \leqslant m-1$. Hence,

$$
\left.\pi_{m}(A) \approx \pi_{m}(\tilde{A}) \approx H_{m}(\tilde{A}) \approx H_{m}\left(\tilde{A}, \widetilde{f\left(S_{1}^{1} \vee \ldots \vee\right.} S_{\mu}^{1}\right)\right)
$$

is a f.g. $Z \pi_{1}$-module (see the proof of Theorem $A$ of [13]) 
Since $\pi_{2}(W-X) \rightarrow \pi_{2}(V-X)$ is the zero map, the map $\pi_{2}(A) \rightarrow \pi_{2}(V-X)$ is surjective. Therefore, $\pi_{2}(V-X)$ is a f.g. $Z \pi_{1}$-module since $\pi_{2}(A)$ is f.g. by Sublemma A.

Finally, the second part of the lemma follows from this fact and the homotopy sequence of the pair $(\widetilde{V-X}, \overrightarrow{\partial V})$.

Remark 2. From page 21 of [15], the cellular chain complex $C_{*}^{c}(\tilde{A})$ is chain complex of $\mathrm{f}$.g. free $\Lambda$-modules. If $\pi_{1}(A)$ is a $\mathrm{f} . \mathrm{g}$. abelian group, then $\Lambda$ is a Noetherian ring (Prop. 1 (c), p. 154, [6]). Therefore, Sublemma $\mathrm{A}$ is also true if $\pi_{1}(A)$ is f.g., abelian (see pp. 71, 22 of [6]).

LEMMA 3. Suppose that $X$ has arbitrarily small neighborhoods $V$ 's as in Lemma 1 such that $\pi_{q}(\partial V)=\pi_{q}(V-X)=\pi_{q}\left(S^{1}\right)$ for $q \leqslant m-1$, where $m \leqslant\left[\frac{1}{2} n\right]$. If $X$ is globally homotopically unknotted, then $\pi_{m}(V-X)$ and $\pi_{m}(V-X, \partial V)$ are $f . g . \Lambda$-modules.

Proof. Let $V$ be such a neighborhood of $X$. Choose another $W$ such that $\pi_{m}(W-X) \rightarrow \dot{\pi}_{m}(V-X)$ is the zero map. The existence of such $W$ is from the globally $m$-LC property of $X$.

The proof now proceeds exactly the same as that one of Lemma 2 . We have to show $\pi_{q}(V-X, W-X)=0$ for all $q, 2 \leqslant q \leqslant m-1$ in order to have $\pi_{m}(A)$

$\approx \pi_{m}(\tilde{A}) \approx H_{m}(\tilde{A})$ to be a f.g. $\Lambda$-module.

Indeed, we have

(a) $V-X=(W-X) \cup A$ and $A \cap W-X=\partial W$ is collared in $A$ and $W-X$,

(b) inclusions map of $\partial W$ into $A, W-X, V-X$ induce isomorphisms on fundamental groups,

(c) $\pi_{q}(W-X, \partial W)=0$ for $q \leqslant m-1, \pi_{q}(A, \partial W)=0$ for $q \leqslant 1$.

Theorem 9.3.5 in [12] gives

$$
\pi_{q}(A, \partial W) \approx \pi_{q}(V-X, W-X) \text { for } q \leqslant m-1 .
$$

Moreover, from the homotopy sequence of the pair $(V-X, W-X)$ and the hypothesis for $V, W$, it follows $\pi_{q}(V-X, W-X)=0, q \leqslant m-1$. The proof of Lemma 3 is finished.

LEMMA 4. If $X$ is globally homotopically unknotted, then $X$ has arbitrarily small neighborhoods $V$ 's as in Lemma 1 satisfying

(a) $\pi_{q}(V-X, \partial V)=0$ for $q \leqslant 2$,

(b) $\pi_{q}(V-X)=\pi_{q}\left(S^{1}\right)$ for $q \leqslant 2$.

Proof. (i) To show (a) $)_{2}$ : Assume that the set $\left\{x_{1}, \ldots, x_{m}\right\}$ generates the $A$-module $\pi_{2}(V-X, \partial V)$. Let $f:\left(D^{2}, S^{1}\right) \rightarrow(V-X, \partial V)$ be a nice PL (or DIFF)-embedding which represents $x_{1} . W$ is obtained from $V$ by carving an open regular neighborhood of $f\left(D^{2}\right)$ in $V-X$. Then $V=W \cup(n-2)$-handle and $V=W \cup D^{n-2}$ is an $(n-2)$-disk traverse to $f\left(D^{2}\right)$. Since $n-2 \geqslant 3$, it is easy to show that $W$ satisfies the conclusion of Lemma 1 , and the $A$-module $\pi_{2}(W-X, \partial W)$ generated by the images of $x_{2}, \ldots, x_{m}$. Inductively, we shall cancell all $x_{i}, i=1,2, \ldots, m$ to obtain a desired neighborhood of $X$ in $V$. (ii) Sublemm a B. For every neighborhood $V$ of $X$ enjoying (*) and (a) , there exists a neighborhood $U$ of $X$ in Int $V$ such that $U$ satisfies $(*),(\mathrm{a})_{2}$ and (b) ${ }_{2}$.

Proof. Let $W$ be a neighborhood of $X$ in Int $V$ satisfying (*), (a) $)_{2}$ and $\operatorname{Im}\left(i_{\text {抹 }}: \pi_{2}(W-X) \rightarrow \pi_{2}(V-X)\right)=0$. Let $A=V$-Int $W$. Suppose that $\left\{y_{1}, \ldots, y_{p}\right\}$ generates the $A$-module $\pi_{2}(W-X)$ (Lemma 2). Let $f: S^{2} \rightarrow W-X$ represent $y_{1}$ Then, there is an extension $f$ of $f$ over $D^{3}, f:\left(D^{3}, S^{2}\right) \rightarrow(V-X, W-X)$

On the other hand, by the homotopy excision theorem (Th. 9.3.5, [12]), the map $i_{\#}: \pi_{3}(A, \partial W) \rightarrow \pi_{3}(V-X, W-X)$ is surjective. Thus, there is a map $g:\left(D^{3}, S^{2}\right) \rightarrow(A, \partial W)$ such that $g \simeq f$ in $(V-X, W-X)$; in particular, $\left[g \mid S^{2}\right]=[f]$ in $\pi_{2}(W-X)$ up to the action of $\pi_{1}(W-X)$

Furthermore, we can assume that $g$ is a nice PL-embedding by Theorem 4.7.1 in [5] or Lemma 4.8 in [11].

Let $U_{1}$ denote $W \cup N\left(g\left(D^{3}\right), A\right)$. It can be shown easily that $U_{1}$ also satisfies (*) and $\pi_{2}\left(U_{1}-X\right) \cong \pi_{2}(W-X) / \Lambda$. [ $\left.y_{1}\right]$. Thus, $\pi_{2}\left(U_{1}-X\right)$ is generated by the images of $y_{2}, \ldots, y_{m}$.

In order to prove that $U_{1}$ satisfies (a) $)_{2}$ we assume that $U_{1}$ contains a copy of $W$ such that $B=U_{1}$-Int $W=\partial W \times[0,1] \cup$ (3-handle). Since $n \geqslant 6$, we can prove that $\pi_{2}\left(U_{1}-X, \partial U_{1}\right)=0$ (we will give in detail a proof for the general case in Lemma 5).

Now, the map $i_{\text {\#\# }}: \pi_{2}\left(U_{1}-X\right) \rightarrow \pi_{2}\left(U_{1}-X\right) \rightarrow \pi_{2}(V-X)$ is still trivial. Hence, we can successively kill all generators of $\pi_{1}\left(U_{1}-X\right)$ to obtain a desired neighborhood $U$ of $X$ in $V$.

LEMMA 5. If $X$ is globally homotopically unknotted, then $X$ has arbitrarily small simply connected neighborhoods $V^{\prime}$ 's in $S^{n}$ satisfying $(*)$ and

(a) $\pi_{q}(V-X, \partial V)=0$ for $q<\left[\frac{1}{2} n\right]$,

(b) $\pi_{q}(V-X)=\pi_{q}\left(S^{1}\right)$ for $q \leqslant\left[\frac{1}{2} n\right]-1$,

(c) $\pi_{q}(V-X)=\pi_{q}\left(S^{1}\right)$ for $q<\left[\frac{1}{2} n\right]-1$.

Proof. Let $(\mathrm{a})_{m},(\mathrm{~b})_{m},(\mathrm{c})_{m}$ denote the conclusion (a), (b), (c) of the lemma for $q \leqslant m$. Assume $n=2 k+2$ or $n=2 k+1$. We will prove by induction on $m \leqslant k-1$, starting with $(\mathrm{a})_{2},(\mathrm{~b})_{1},(\mathrm{c})_{1}$ by Lemma 4 ; first, killing $\pi_{m}(V-X)$; next, $\pi_{m}(\partial V)$; and finally, $\pi_{m+1}(V-X, \partial V)$.

Step $\mathrm{I}$. Assume that $X$ has arbitrarily small neighborhoods satisfying (*) $(\mathrm{a})_{m},(\mathrm{~b})_{m-1}$ and $(\mathrm{c})_{m-1}$. Given such a neighborhood $V$, we will construct a neighborhood $U$ of $X$ in $V$ satisfying (*) (a) $)_{m},(b)_{m}$ and $(c)_{m-1}$.

Let $W$ be a neighborhood of $X$ in $V$ satisfying (*), (a) $)_{m},(\mathrm{~b})_{m-1}$ and $(\mathrm{c})_{m-1}$

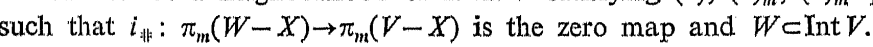

By Lemma 3 , we can assume that $\left\{x_{1}, \ldots, x_{\alpha}\right\}$ generates the $\Lambda$-module $\pi_{m}(W-X)$. A similar argument to that in Lemma 4 , there is a nice PL-embedding $f:\left(D^{m+1}, S^{m}\right)$ $\rightarrow(A, \partial W)$ such that the homotopy class $\left[f \mid S^{m}\right]$ is also a generator of $\Lambda\left[x_{1}\right]$, where $A=\widetilde{V-W}$.

Denote $U^{\prime}=W \cup N\left(f\left(D^{m+1}\right), A\right)$. It is clear that $U^{\prime}$ enjoys the property $\left(^{*}\right)$ of Lemma 1.

5 - Fundamenta Mathematicae CII 
Property (a) $)_{m}$ : We can assume that $W \subset \operatorname{Int} U^{\prime}$ such that

$$
B=U^{\prime}-\text { Int } W=(\partial W \times[0,1]) \cup(m+1) \text {-handles . }
$$

Theorem 9.3 .5 of [12] shows that $i_{\#}: \pi_{q}\left(U^{\prime}-X, B\right) \rightarrow \pi_{q}(W-X, \partial W)$ is an isomorphism for all. $q \leqslant m$, since for $q \leqslant m, \pi_{q}\left(\partial W \cup D^{m+1}, \partial W\right)=0(m \geqslant 2)$, and $\pi_{q}(W-X, \partial W)=$ for all $q \leqslant m$.

Moreover, from Hurewicz's theorem, duality of handle decomposition [5] it follows that $\pi_{a}\left(B, \partial U^{\prime}\right)=0$ for all $q \leqslant m$, since $m<n-(m+1)$.

Now, from this fact and the homotopy sequence of the triple $\left(U^{\prime}-X, B, \partial U^{\prime}\right)$ we can conclude that

$$
\pi_{q}\left(U^{\prime}-X, \partial U^{\prime}\right)=0 \text { for all } q \leqslant m .
$$

PROPERTY $(\mathrm{c})_{m-1}$. Since $U^{\prime}-X$ has the homotopy type of $(W-X) \cup_{f} D^{m+1}$, $\pi_{q}\left(U^{\prime}-X\right)=\pi_{q}\left(S^{1}\right)$ for all $q \leqslant m-1$. Hence, we still have $(\mathrm{a})_{m-1}$ and $(\mathrm{c})_{m-1}$.

Finally, $\pi_{m}\left(U^{\prime}-X\right) \approx \pi_{m}(W-X) / \Lambda$. $\left[x_{1}\right]$. Hence $\pi_{m}\left(U^{\prime}-X\right)$ is generated by the image of $x_{2}, \ldots, x_{\alpha}$. The proof will be finished after killing $x_{2}, \ldots, x_{\alpha}$ successively.

Step II. Assume that $X$ has arbitrarily small neighborhoods satisfying (*), (a) (b) $m$ and (c) $)_{m-1}$. Given such a neighborhood $V$, we will construct a neighborhood $U$ of $X$ in $V$ satisfying $(*),(\mathrm{a})_{m},(\mathrm{~b})_{m}$ and $(\mathrm{c})_{m}$.

Assume that $\left\{y_{1}, \ldots, y_{\beta}\right\}$ generates the $\Lambda$-module $\pi_{m}(\partial V)$. Since $\pi_{m}(V-X)=0$, there is a nice PL-embedding $f:\left(D^{m+1}, \dot{S}^{m}\right) \rightarrow(V-X, \partial V)$ such that $\left[f \mid S^{m}\right]=y_{1}$ Let $U^{\prime}=V-\operatorname{Int} V\left(f\left(D^{m+1}\right), V-X\right)$, then $U^{\prime}$ is a better neighborhood of $X$ in $V$ Indeed,

(i) Clearly, $U^{\prime}$ enjoys the property (*).

(ii) $\pi_{q}\left(U^{\prime}-X\right) \approx \pi_{q}\left(S^{1}\right)$, for $q \leqslant m \leqslant k-1$, since $(V-X) \cup(n-(m+1))$-handle.

(iii) We can assume that $U^{\prime} \subset \operatorname{Int} V . B$ denotes $V$-Int $U^{\prime}$. The homology sequence of $\left(\overparen{V-X}, \widetilde{B}, \overparen{\partial V)}\right.$ gives $H_{q}(\overparen{V-X}, \widetilde{B})=0$ for $q \leqslant m$, since $H_{q}(\widetilde{V-X}, \overparen{\partial V)}=0$ and $H_{q-1}(\widetilde{B}, \widetilde{\partial V})=0$ for all $q \leqslant m$. Now, the excision theorem and Hurewicz's theorem give

$$
0=H_{\dot{q}}(\widetilde{V-X}, \widetilde{B})=H_{q}\left(\widetilde{U^{\prime}-X}, \widetilde{\partial U^{\prime}}\right)=\pi_{q}\left(U^{\prime}-X, \partial U^{\prime}\right) \text { for all } q \leqslant m .
$$

(iv) $\pi_{m}\left(\partial U^{\prime}\right) \approx \pi_{m}(\partial V) / \Lambda .\left[y_{1}\right]$.

We can proceed finitely many times this handle trading process to obtain a desired neighborhood $U$.

Step III. This step is exactly the same as Step II, since we also have $\pi_{m+1}(V-X, \partial V)$ is f.g. $A$-module by Lemma 3 .

In order to complete the proof of the lemma, we need to prove $(b)_{k}$, when $n=2 k+2$. However, the proof of Step I also works for this case.

LEMMA 6. If $X$ is glcbally homotopically, unknotted, then the end of $S^{n}-X$ is tame.
Proof. Case $1(n=2 k+2 \geqslant 6)$. Let $V$ be a neighborhood $X$ satisfying the conclusion of Lemma 5 . Then

Claim. (i) $H_{q}(V-X, \partial V)=0$ for $q \neq k+1$,

(ii) $H_{k+1}(V-X, \partial V)$ is a projective, f.g. A-module,

(iii) $H^{k+2}((V-X, \partial V) ; G)=0$ for every $\Lambda$-module $G$.

Hence, $V-X$ is finitely dominated by the theorem in [14].

Let $W$ be a neighborhood of $X$ in Int $K$ satisfying Lemma 5 and the map $i_{\text {非 }}: \pi_{k+1}(W-X) \rightarrow \pi_{k+1}(V-X)$ is trivial. Then, from the homotopy sequence of the pair $(\overline{V-X}, \overparen{W-X})$, the Hurewicz theorem, it follows that

$$
H_{k+1}(\widetilde{V-X}) \approx H_{k+1}(\overparen{V-X}, \overparen{W-X}) \approx H_{k+1}(\tilde{A}, \widetilde{\partial W}) \text { (excision), }
$$

which is a f.g. $\Lambda$-module since $(A, \partial W)$ is a pair of finite CW-complexes. That proves a half of (ii).

Let $A=V$-Int $W$. Then, the connectivity of $V-X, W-X$ and the homotopy excision theorem (Th. 9.3.5, [12]) show that $\pi_{\dot{q}}(A, \partial W)=0$ for $q \leqslant k$.

Now, by the handle cancellation theorem (p. 246, [5]), we have a handle decomposition of $A$ rel. $\partial W$ having no handles of index $\leqslant k$. Hence, there is dually a handle decomposition of $A$ rel. $\partial V$ having no handles of index $\geqslant n-k=k+2$.

Using a nested sequence of such nice neighborhoods and glueing a sequence of nice Morse functions, we can obtain a handle decomposition of $V-X$ rel. $\partial V$ having no handles of index $\geqslant k+2$. Hence, the cellular chain complex of the pair $(V-X, \partial V)$ has the property that

Therefore,

$$
C_{q}^{c}(\widetilde{V-X}, \widetilde{\partial V})=0 \quad \text { for all } q \geqslant k+2 .
$$

and

$$
H_{q}(\widetilde{V-X}, \widetilde{\partial V})=0 \quad \text { for all } q \geqslant k+2 \text {, }
$$

$$
H^{q}(\widetilde{V-X}, \overrightarrow{\partial V} ; G)=0 \text { for all } q \geqslant k+2,
$$

and $\Lambda$-module $G$. That proves (i) and (iii).

Now, from Theorem 8 (iii) of [14], it follows that $H_{k+1}(\overparen{V-X}, \widetilde{\partial V})$ is a projective A-module.

This completes the proof of (ii).

Case $2(n=2 k+1 \geqslant 7)$. First, similar to the proof of Sublemma B, we can show that $X$ has arbitrarily small neighborhoods $V$ 's that

(i) $\pi_{q}(V-X)=\pi_{u}\left(S^{1}\right)$ for $q \leqslant k$,

(ii) $\pi_{l}(V-X, \partial V)=0$ for $q \leqslant 2$.

Since $\pi_{k}(V-X)$ is finitely generated, the homotopy excision theorem (Th. 9.3.5 [12]) also works in this case (even after trading $(k+1)$-handles since $2(k-1) \geqslant k+1$ for $k \geqslant 3)$; and $\pi_{q}(V-X)(q \leqslant k-1)$ is unchanged after adding $(k+1)$-handles. 
Secondly, using a proof similar to that for Case 1, we can obtain a handle decomposition of $V-X$ rel. $\partial V$ ( $V$ satisfying (i) and (ii); above) having no handles of index $\geqslant k+1$.

Finally, it will be prowed that $V-X$ is finitely dominated.

THEOREM I. Let $X$ be a compactum in $S^{n}, n \geqslant 6$, having the shape of the codimension-2 sphere $S^{n-2}$. If $X$ is globally homotopically unknotted in $S^{n}$, then there is a locally flat $(n-2)-$ sphere $\Sigma$ in $S^{n}$ such that $S^{n}-X \approx S^{n}-\Sigma$.

Proof [3]. By Lemma 6, the Main Theorem of [11] shows that the end of $S^{n}-X$ has a collar, i.e. $X$ has a PL-neighborhood $V$ such that

$$
V-X \approx \partial V \times[0,1) \text {. }
$$

Using this product structure of $V-X$ and the globally homotopic unknottedness of $X$, we can show that

$$
\pi_{q}(\partial V)=\pi_{q}(V-X)=\pi_{q}\left(S^{1}\right) \text { for } \quad q \leqslant\left[\frac{1}{2} n\right] .
$$

Let $D^{2}$ be the image of a proper embedding of the 2 -cell into $V$ such that $\partial D^{2}$ represents the generator of $\pi_{1}(\partial V)=Z$, and $D^{2} \times D^{n-2}$ the regular neighborhood of $D^{2}$ in $V$.

If $B=V\left(D^{2} \times \operatorname{Int} D^{n-2}\right)$, then it is clear that $\pi_{q}(\partial B)=0$ for $q \leqslant\left[\frac{1}{2} n\right]$. Hence $\partial B \simeq S^{n-1}$ by Poincaré duality, and thus $\partial B \approx S^{\text {PL }}$.

Therefore, $B$ is the Pl $n$-ball and $V=B \cup(n-2)$-handle.

Moreover, by Levine's theorem [7], the attach sphere $0 \times D^{n-2}$ of this handle is unknotted in $\partial B$ since

$$
\pi_{q}\left(\partial B-0 \times \partial D^{n-2}\right) \approx \pi_{q}(\partial V)=\pi_{q}\left(S^{1}\right) \text { for } q \leqslant\left[\frac{1}{2} n\right] .
$$

Hence, it is the trivial $(n-2)$-handle, i.e. $h: \stackrel{\text { PL }}{V \approx D^{2} \times S^{n-2}}$; and $\Sigma$ is the image in $S^{n}$ of $0 \times S^{n-2}$ under $h^{-1}$

LEMMA 7. Let $\left\{A_{i}\right\} \stackrel{\alpha}{\rightarrow}\left\{B_{i}\right\} \stackrel{\beta}{\rightarrow}\left\{C_{i}\right\} \stackrel{\gamma}{\rightarrow}\left\{D_{i}\right\} \stackrel{\delta}{\rightarrow}\left\{E_{i}\right\}$ be an exact sequence of inverse sequences of abelian groups. If the bounding maps of $\left\{B_{i}\right\}$ are isomorphisms, and $\left\{A_{i}\right\},\left\{D_{i}\right\},\left\{E_{i}\right\}$ are stable, then $\left\{C_{i}\right\}$ is stable.

Proof. Without loss of generality we can assume that $\left\{A_{i}\right\},\left\{D_{i}\right\}$ and $\left\{E_{i}\right\}$ are constant sequences (Siebenmann's terminology [11]).

Consider the diagram

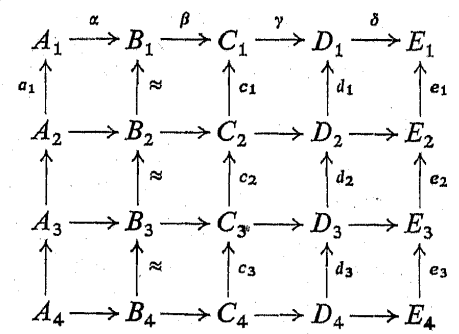

(i) $c_{1} \mid \operatorname{Im} c_{2}: \operatorname{Im} c_{2} \rightarrow \operatorname{Im} c_{1}$ is surjective. It suffices to show that for every $z_{2} \in c_{2}$, there exists $z_{3} \in C_{3}$ such that $c_{1} c_{2}\left(z_{3}\right)=c_{1}\left(z_{2}\right)$.

Since $\left\{D_{i}\right\}$ is constant, there is $w_{4} \in D_{4}$ such that

$$
d_{1} d_{2} d_{3}\left(w_{4}\right)=d_{1} \gamma\left(z_{2}\right)=\gamma c_{1}\left(z_{2}\right) \text {. }
$$

Let $z_{3}^{\prime} \in C_{3}$ such that $\gamma\left(z_{3}^{\prime}\right)=d_{3}\left(w_{4}\right)\left(\delta d_{3}\left(w_{4}\right)=0\right.$ by stability of $\left.\left\{E_{i}\right\}\right)$. Then, $\gamma c_{1} c_{2}\left(z_{3}^{\prime}\right)=d_{1} d_{2} \gamma\left(z_{3}^{\prime}\right)$. Hence

$$
\gamma\left(c_{1}\left(z_{2}\right)-c_{1} c_{2}\left(z_{3}^{\prime}\right)\right)=0 \in D_{1} .
$$

From the exactness of the sequence, there is $y_{3} \in B_{3}$ such that $\beta b_{1} b_{2}\left(y_{3}\right)$ $=c_{1}\left(z_{2}\right)-c_{1} c_{2}\left(z_{3}^{\prime}\right)$.

Let $z_{3}=z_{3}^{\prime}+\beta\left(y_{3}\right)$. Then, $z_{3}$ is a desired element.

(ii) To show $c_{2} \mid \operatorname{Im} c_{3}$ is injective. It suffices to show that if $z_{4} \in C_{4}$ such that $c_{2} c_{3}\left(z_{4}\right)=0$, then $c_{3}\left(z_{4}\right)=0$.

Now, by stability of $\left\{D_{i}\right\}$, there is $y_{3} \in B_{3}$ such that $\beta\left(y_{3}\right)=c_{3}\left(z_{4}\right)$. Then from the exactness, there is an element $x_{2} \in A_{2}$ such that $\alpha\left(x_{2}\right)=b_{2}\left(y_{3}\right)$.

From the stability of $\left\{A_{i}\right\}$, there is $x_{4} \in A_{4}$ such that $\alpha\left(x_{4}\right)=b_{3}^{-1}\left(y_{3}\right)$.

Hence, $c_{3}\left(z_{4}\right)=\beta b_{3} \alpha\left(x_{4}\right)=c_{3} \beta \alpha\left(x_{4}\right)=0$.

Lemma 8. Let $X$ be a compactum in an orientable closed manifold $M$ such that $X$ has the shape of a codimension-2 finite complex. If $X^{*}$ is globally 1 -alg then the end of $M-X$ is stable.

Proof. By Lemma 1 of [8], there is a nested sequence of neighborhoods $\left\{V_{i}\right\}$ of $X$ such that the sequences $\left\{H_{2}\left(V_{i}\right)\right\}$ and $\left\{H_{1}\left(V_{i}\right)\right\}$ are constant. Furthermore,

$$
i_{*}: H_{j}\left(V_{i+1}, V_{i+1}-X\right) \stackrel{\approx}{\rightarrow} H_{j}\left(V_{i}, V_{j}-X\right)
$$

by excision theorem.

Hence, by Lemma 7 the sequence $\left\{H_{1}\left(V_{i}-X\right)\right\}$ is stable.

Finally, using the globally 1 -alg property and chasing diagram as in [3] or [8], we can show that the end is stable and $\pi_{1}(\varepsilon)=\lim _{\rightarrow \rightarrow} H_{1}\left(V_{i}-X\right)$ is a finitely represented abelian group.

Trreorim II. Let $X$ be a compactum in an orientable closed manifold $M^{n}, n \geqslant 6$, such that $X$ has the shape of the $(n-2)$-torus. If $X$ is globally homotopically unknotted, then $M-X$ has a collar at the end 8 .

Proof. The proof of Lemmas 1-6 remains valid in this case. It follows that the end $a$ is tame.

An easy diagram chase proves that the following sequence is exact

$$
Z \rightarrow \lim _{i} H H_{1}\left(V_{l}-X\right) \rightarrow \lim _{i} H_{1}\left(V_{i}\right) \rightarrow 0
$$

where $\left\{V_{i}\right\}$ is a suitable nested sequence of neighborhoods of $X$.

Since $\lim H_{1}\left(V_{l}\right)=H_{1}\left(T^{n-2}\right)=Z^{n-2}, \pi_{1}(\varepsilon)$ is finitely generated, abelian. 
Now, let $V$ be a neighborhood of $X$ satisfying (a) ${ }_{2}$ and $(b)_{2}$, then from the homotopy excision theorem and engulfing theorem, it is easy to prove that $\pi_{2}(V)=0$, and $\pi_{1}(V) \approx \pi_{1}\left(T^{n-2}\right)=Z^{n-2}$. Then, the following diagram

$$
\begin{gathered}
0=\pi_{2}(V) \rightarrow \pi_{2}(V, V-X) \rightarrow \pi_{1}(V-X) \rightarrow \pi_{1}(V) \rightarrow 0 \\
\downarrow \\
H_{2}(V) \rightarrow H_{2}(V, V-X) \rightarrow H_{1}(V-X) \rightarrow H_{1}(W) \rightarrow 0 \\
2^{\downarrow} \\
Z
\end{gathered}
$$

shows that $\pi_{2}(V, V-X)=Z$ and $\pi_{1}(V-X) \approx Z^{n-1}$.

Since the group $\widetilde{K}_{0} \pi_{1}(\varepsilon)=0$ (see [11] and III. 11.3 of [2]), it follows that the end of $M-X$ has a collar from the main theorem of [11].

Definitron. Let $\Omega^{n}$ be an open manifold with one end $\varepsilon . \varepsilon$ is said to be $q$ th $H(h)$-stable if the inverse system $\left\{\pi_{q}\left(V_{j}\right), i_{\#}\right\}\left(\left\{H_{q 1}\left(\tilde{V}_{j}\right), i_{*}\right\}\right)$ is stable, where $\left\{V_{j}\right\}$ is a cofinal sequence of neighborhoods of $\varepsilon . \pi_{q}(\varepsilon)$ denotes the qth-homotopy group of the end, $\lim \pi_{q}\left(V_{j}\right)$, if any.

A diagram

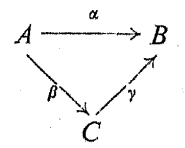

is $q$-commutative up to homotopy, if $\alpha\left|A^{(q)} \simeq \gamma \beta\right| A^{(q)}$, where $A^{(q)}$ is the qth-skeleton of the CW-complex $A$.

$\varepsilon$ is $q$-equivalent to a finite complex $K$, if there is a nested sequence of neighborhoods $\left\{V_{j}\right\}$ of $\varepsilon$ and maps $f_{j}: K \rightarrow V_{j}$ and $g_{j}: V_{j} \rightarrow K$ such that the following diagram is $q$-commutative, up to homotopy

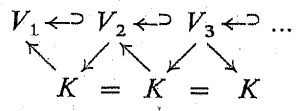

Remark 3. Since $(k+1)$-skeleton of a CW-complex determines its $q$ th homotopy group for all $q \leqslant k$, the end $\varepsilon$ is $q$-stable, $q \leqslant k$ if it is $(k+1)$-equivalent to a complex $K$ (similar to Lemma 1, [8].)

Similarly, $H_{q}(\tilde{V})$ is f.g. $(q \leqslant k+1)$, if the end is $(k+1)$-equivalent to a finite complex $K$ with $\pi_{1}(K)$ f.g., abelian.

Definition. A finite complex $K$ is $m$-admissible if $\pi_{1}(K)$ is finitely generated abelian and $\pi_{q}(K)$ is a f.g. $Z \pi_{1}(K)$-module for $2 \leqslant q \leqslant m$.

THEOREM III. Let $\Omega^{n}$ be an open PL (or DIFF) manifold with one end \& $(n \geqslant 6)$. If $\varepsilon$ is $\left[\frac{1}{2}(n+1]\right.$-equivalent to an $\left[\frac{1}{2} n\right]$-admissible finite complex $K$, then there is an obstruction $\sigma \in \widetilde{K}_{0} \pi_{1}(K)$ which vanishes if and only if $\Omega$ has a collar at $\varepsilon$.
COROLLARY TO THEOREM III. Let $M^{n-2}$ be a simply connected codimension-2 submanifold of a closed PL (or DIFF)-manifold $N^{n}(n \geqslant 6)$. If the end of $N-M$ is $\left[\frac{1}{2}(n+1)\right]$-equivalent to $S^{1} \times M$, then $N-M$ has a collar at its end.

Outline the proof of Theorem III. Define

Statement $(a)_{j}=\varepsilon$ has arbitrarily small neighborhoods $V$ such that $\pi_{q}(V, \partial V)=0, q \leqslant j$

Statement $(\mathrm{b})_{j}=\varepsilon$ has arbitrarily small neighborhoods $V$ such that $\pi_{q}(\varepsilon) \stackrel{\approx}{\rightarrow} \pi_{q}(V), q \leqslant j$.

It will be proved by induction on $j \leqslant k-1$ starting with $j=1\left((\mathrm{a})_{1},(\mathrm{~b})_{1}\right.$ exist by [11]).

It follows easily from Remark 3 that if $V$ satisfies $(a)_{1}$ and $(b)_{1}$, then $\pi_{2}(V)$ and $\pi_{2}(V, \partial V)$ are f.g. $Z \pi_{1}(\varepsilon)$-module. Hence we can obtain (a) $)_{2}$ by carving 2 -handles.

To obtain (b) $)_{2}$ from $\left\{(\mathrm{a})_{2}+(\mathrm{b})_{1}\right\}$. Choose $W \subset \operatorname{Int} V$ satisfying $(\mathrm{a})_{2}$ and $(\mathrm{b})_{1}$ and $\operatorname{Im}\left(i_{\text {非: }} \pi_{2}(W) \rightarrow \pi_{2}(V)\right)$ is $\pi_{2}(\varepsilon)$ :

Since $\pi_{2}(W)$ is f.g., and $Z \pi_{1}(\varepsilon)$ is Noetherian, ker $i_{\text {\# }}$ is f.g. Then, the argument of Sublemma B allows to add 3-handles (lying $V$-Int $W$ ) to $W$ to obtain (b) ${ }_{2}$. To obtain $(\mathrm{a})_{3}$ : Start with $(\mathrm{a})_{2}$ and $(\mathrm{b})_{2}$.

The Hurewicz theorem and $(\mathrm{a})_{2}$ shows that $\pi_{3}(V, \partial V) \approx H_{3}(\widetilde{V}, \widetilde{\partial V})$; but the latter is f.g., since $H_{3}(\tilde{V})$ and $H_{2}(\partial V)$ are f.g. by Remark 2 and Remark 3. Hence, we can obtain (a) $)_{3}$ and so on. Finally, as in Lemma 6 we can obtain (b) $)_{k}$ and $(a)_{k-1}$. The proof will complete in routine.

Proof of the corollary. It suffices to mention that $\pi_{1}\left(S^{1} \times M\right) \pi_{1}\left(S^{1}\right)=Z$ and $\pi\left(S^{1} \times M\right)=\pi_{q}(M)$ f.g. abelian for all $q$ by Corollary 9.6 .16 in [12]

\section{References}

[1] K. Borsuk, Concerning homotopy property of compacta, Fund. Math. 62 (1968), pp. 223-254.

[2] M. M. Cohen, A Course in Simple Homotopy Theory, Springer Verlag, New York 1970.

[3] R. J. Daver'man, Homotopy classification of complements of locally flat codimension-two spheress (to appear).

[4] J. G. Hollingsworth and T. B. Rushing, Homotopy characterization of weakly flat codimension-2 spheress (to appeatr).

[5] J. F. P. Hudson, Pieces'se' Linear Topology, W. A. Benjamin, New York 1969.

[6] J. Lambeck, Lectures' on Rings' ant Modules, Blaisdell, Massachusettes 1966.

[7] J. Lovine, Unknotting spheres in codimension two, Topology 4 (1965), pp. 9-16.

[8] V. 'T. Liom, Certuin continua in S" of the same shape have homeomorphic complements, Trans. Amer. Math. Soc, 218 (1976), p1). 207-217.

[9] S. Mardešić and J. Segal, Shape of e'ompacta and ANR-systems, Fund. Math. 72 (1971), pp. 47.59 .

[10] … Liquivalence of the Borsuk and the ANR-systems upproach to shapes, Fund. Math. 72 (1971), pp. 6108.

[11] L. C. Siebenmann, The obstruction to finding a boundary for an open manifold of dimension greater than five, Ph. D. Dissertation, Princeton Univ. 1965. 
[12] E. H. Spanier, Algebraic Topology, McGraw Hill, New York 1966.

[13] C. T. C. Wall, Finiteness conditions for CW-complexes, Ann. of Math. 81 (2) (1965), pp. $56-69$.

[14] - Finiteness conditions for CW-complexes II, Royal Soc. Proc., Ser. A, 295 (1966), pp. 129-139.

[15] - Surgery on Compact Manifolds, Academic Press, New York 1970.

UNIVERSITY OF GEORGIA

\section{On decompositions of hereditarily unicoherent continua}

Eldon J. Vought (Chico, Calif.)

Abstract. Charatonik has defined a monotone upper semi-continuous decomposition of a continuum to be admissible if the layers of its irreducible subcontinua an decomposition of a conof the decomposition. He has constructed an admissible of continua, e.g. $\lambda$-dendroids, is unique and minimal with decomposition which, for many classes connected quotient space. This paper studies hereditarily respect to having an hereditarily arcwise of closed separators (closed sets equivalent description of Charatonik's decorate the space) with certain properties to obtain an precisely when such a Charatonik's decomposition. This viewpoint enables one to describe in showing the such a continuum has a non-trivial admissible decomposition. One of the difficulties in showing the equivalence is due to the lack of an adequate description of the layers of an irreducible continuum having non-void interiors. A secondary purpose of this paper is to provide an irreducible tion. A monostratic continuum is one which does not have a non-trivial to provide such a descripand an example is given at the end to show that a $\lambda$-dendroid -rivial admissible decomposition monostratic subcontinua yet not admit an admissible dendroid may have no interior containing void interior.

The study of monotone upper semi-continuous decompositions of continua with "nice" quotient spaces has been undertaken by a large number of authors. Of particular interest are three such works. FitzGerald and Swingle have described in [5] a construction that yields a unique decomposition which is the finest possible with respect to having a semi-locally connected quotient space. By a different technique in [6], McAuley, using closed separators, has constructed a decomposition equivalent to the one above. And Charatonik in [3] has given a decomposition which, for certain continua, e.g., $\lambda$-dendroids and atriodic continua, is unique and minimal with respect to having an hereditarily arcwise connected quotient space.

If $I$ is an irreducible continuum there exists a unique minimal monotone upper semi-continuous decomposition whose quotient space is degenerate or an arc [7, p. 10]. The elements of this decomposition are called letyers and Charatonik $[3$, p. 115] has defined a decomposition $D$ of a continuum $M$ to be admissible if

1. $D$ is upper semi-continuous,

2. $D$ is monotone,

3. for every irreducible subcontinuum $I$ in $M$, every layer of $I$ is contained in some element of $D$. 